\title{
Non-uniform in time robust global asymptotic output stability
}

\author{
Iasson Karafyllis* \\ Department of Economics, Division of Mathematics, University of Athens, 8 Pesmazoglou Street, 10559 Athens, Greece
}

Received 24 January 2003; received in revised form 23 March 2004; accepted 11 August 2004

Available online 30 September 2004

\begin{abstract}
The notions of non-uniform in time Robust Global Asymptotic Output Stability and robust forward completeness for timevarying systems are introduced. Necessary and sufficient conditions and Lyapunov-like characterizations are given for these notions.

(ㄷ) 2004 Elsevier B.V. All rights reserved.
\end{abstract}

Keywords: Output stability; Forward completeness; Lyapunov functions; Time-varying systems

\section{Introduction}

The goal of this paper is to introduce and provide characterizations for the notions of non-uniform in time Robust Global Asymptotic Output Stability (RGAOS) and Robust Forward Completeness (RFC). Non-uniform in time asymptotic stability is a wellknown notion that appears only in time-varying systems and recently this notion was exploited for the robust stabilization of control systems by means of time-varying feedback (see [5-10]).

Characterizations of Robust Forward Completeness (RFC) for general dynamical systems were given in the pioneering paper [1] under the assumption that the right-hand side of the differential equation is locally

\footnotetext{
* Corresponding author. Tel.: +21-09-942-608; fax: +21-09-942611.

E-mail address: iasonkar@e-free.gr (I. Karafyllis).
}

Lipschitz. The results of [1] were based on a primary result obtained in [12], which holds for differential equations with locally Lipschitz dynamics. However, for time-varying systems the requirement that the dynamics are locally Lipschitz with respect to time is a restrictive requirement. Moreover, it is clear that if there exists an equilibrium point, then the general estimates of the solution of a forward complete dynamical system obtained in [1] are conservative. We also notice the Lyapunov characterization of forward completeness given in [11] for time-varying systems without inputs. In this paper, we intend to relax the requirement of Lipschitz continuity with respect to time for systems with inputs and provide less conservative estimates for forward complete dynamical systems with an equilibrium point. Particularly, we consider the class of systems:

$$
\begin{aligned}
& \dot{x}=f(t, x, d), \\
& x \in \mathfrak{R}^{n}, \quad t \geqslant 0, \quad d \in D .
\end{aligned}
$$


We assume that $D \subset \mathfrak{R}^{m}$ is a non-empty compact set and that $f(t, 0, d)=0$, for all $(t, d) \in \mathfrak{R}^{+} \times D$. Furthermore, we assume that the vector field $f: \mathfrak{R}^{+} \times$ $\Re^{n} \times D \rightarrow \Re^{n}$, satisfies the following conditions:

1. The function $f(t, x, d)$ is measurable in $t$, for all $(x, d) \in \Re^{n} \times D$.

2. The function $f(t, x, d)$ is continuous in $d$, for all $(t, x) \in \mathfrak{R}^{+} \times \mathfrak{R}^{n}$.

3. The function $f(t, x, d)$ is locally Lipschitz with respect to $x$, uniformly in $d \in D$, in the sense that for every bounded interval $I \subset \mathfrak{R}^{+}$and for every compact subset $S$ of $\mathfrak{R}^{n}$, there exists a constant $L \geqslant 0$ such that

$|f(t, x, d)-f(t, y, d)| \leqslant L|x-y|$,

$\forall t \in I, \quad \forall(x, y) \in S \times S, \quad \forall d \in D$.

In Section 2 we provide characterizations of the notion of Robust Forward Completeness (RFC) for the class of systems (1.1). Particularly, Theorem 2.2 provides Lyapunov-like characterizations of the notion of robust forward completeness that generalize the corresponding Lyapunov-like characterization given in [1].

The notion of Uniform Robust Global Asymptotic Output Stability (URGAOS) was recently given in $[14,15]$ (where the name ROS was used). New characterizations for versions of this property were given in [4]. Lyapunov characterizations of robust asymptotic output stability notions can be obtained by using the converse Lyapunov Theorem in [16], which also applies to time-varying systems by extending the state vector of the system with the equation $\dot{t}=1$ and assuming Lipschitz continuity of the dynamics with respect to the extended state vector $(t, x)$ (a restrictive requirement as remarked earlier). In this paper, we generalize the notion of Uniform Robust Global Asymptotic Output Stability (URGAOS) to the non-uniform in time case by introducing the notion of (non-uniform in time) Robust Global Asymptotic Output Stability (RGAOS) and we relax the requirement of Lipschitz continuity with respect to time. Particularly, we consider time-varying systems of the form (1.1) with the output map:

$Y=H(t, x)$,

where $H: \mathfrak{R}^{+} \times \mathfrak{R}^{n} \rightarrow \mathfrak{R}^{k}$ is a $C^{0}$ function with $H(t, 0)=0$ for all $t \geqslant 0$. In Section 3 of the present pa- per we give various characterizations for non-uniform in time RGAOS (Propositions 3.2 and 3.6) and links of this notion to (non-uniform in time) Robust Global Asymptotic Stability (RGAS) and RFC (Lemma 3.3 and Proposition 3.5).

Finally, in Section 4 we give a sufficient condition for non-uniform in time RGAOS, which is similar to the corresponding results given in $[4,14,15]$ and same in spirit with analogous results given in $[2,12]$ that provide links between Input-to-State Stability and uniform Robust Global Asymptotic Stability.

Notations. Throughout this paper we adopt the following notations:

* By $M_{D}$ we denote the set of all measurable functions from $\mathfrak{R}^{+}$to $D$, where $D$ is a given compact subset of $\mathfrak{R}^{m}$.

* By $C^{j}(A)\left(C^{j}(A ; \Omega)\right)$, where $j \geqslant 0$ is a nonnegative integer, we denote the class of functions (taking values in $\Omega$ ) that have continuous derivatives of order $j$ on $A$.

* For $x \in \mathfrak{R}^{n}, x^{\prime}$ denotes its transpose and $|x|$ its usual Euclidean norm.

$* \mathrm{~L}_{\mathrm{loc}}^{\infty}$ denotes the set of all measurable functions $u$ : $A \rightarrow \mathfrak{R}^{m}$ that are essentially bounded on every nonempty compact subset of $A$, and $\mathrm{L}^{\infty}(A)$ denotes the set of all measurable functions $u: A \rightarrow \mathfrak{R}^{m}$ that are essentially bounded on $A$.

* By $B[x, r]$ where $x \in \mathfrak{R}^{n}$ and $r \geqslant 0$, we denote the closed sphere in $\mathfrak{R}^{n}$ of radius $r$, centered at $x \in \mathfrak{R}^{n}$.

$* x(t)=x\left(t, t_{0}, x_{0} ; d\right)$ denotes the unique solution of (1.1) at time $t \geqslant t_{0}$ that corresponds to some input $d(\cdot) \in M_{D}$, initiated from $x_{0} \in \mathfrak{R}^{n}$ at time $t_{0} \geqslant 0$ (see [3]).

* $K^{+}$denotes the class of positive $C^{0}$ functions $\phi$ : $\mathfrak{R}^{+} \rightarrow(0,+\infty)$. For the definitions of classes $K, K_{\infty}$ see [12]. By $K L$ we denote the set of all continuous functions $\sigma=\sigma(s, t): \mathfrak{R}^{+} \times \mathfrak{R}^{+} \rightarrow \mathfrak{R}^{+}$ with the properties: (i) for each $t \geqslant 0$ the mapping $\sigma(\cdot, t)$ is of class $K$; (ii) for each $s \geqslant 0$, the mapping $\sigma(s, \cdot)$ is non-increasing with $\lim _{t \rightarrow+\infty} \sigma(s, t)=0$.

* We denote by $\mathrm{E}$ the class of functions $\mu \in$ $C^{0}\left(\Re^{+} ; \mathfrak{R}^{+}\right)$that satisfy $\int_{0}^{+\infty} \mu(t) \mathrm{d} t<+\infty$ and $\lim _{t \rightarrow+\infty} \mu(t)=0$.

* We say that a function $f: \mathfrak{R}^{+} \times \Omega \times \mathfrak{R}^{n} \rightarrow \mathfrak{R}^{k}$ where $\Omega \subset \mathfrak{R}^{m}$ is a compact set, is locally Lipschitz with respect to $x \in \mathfrak{R}^{n}$, uniformly in $\theta \in \Omega$, if 
for every bounded interval $I \subset \mathfrak{R}^{+}$and for every compact subset $S$ of $\mathfrak{R}^{n}$, there exists a constant $L \geqslant 0$ such that

$$
\begin{aligned}
& |f(t, \theta, x)-f(t, \theta, y)| \leqslant L|x-y|, \\
& \forall t \in I, \quad \forall(x, y) \in S \times S, \quad \forall \theta \in \Omega,
\end{aligned}
$$

For the reader's convenience we have collected below some properties concerning functions of the classes $K^{+}, K_{\infty}, K L$, which are repeatedly used in the rest of paper.

Fact I. (Corollary 10 and Remark 11 in Sontag [13]). For each $\gamma \in K_{\infty}$, there exists $\sigma \in K_{\infty}$ such that $\gamma(r s) \leqslant \sigma(r) \sigma(s)$ for all $(r, s) \in\left(\mathfrak{R}^{+}\right)^{2}$.

Fact II. (Fact V in Karafyllis and Tsinias [10]). Let $U \subseteq \mathfrak{R}^{n}$ be a closed set containing $0 \in \mathfrak{R}^{n}$. Let $k$ : $\mathfrak{R}^{+} \times U \rightarrow \mathfrak{R}$ be a $C^{0}$ function with $k(t, 0)=0$ for all $t \geqslant 0$. Then there exist $a \in K_{\infty}$ and $\phi \in K^{+}$such that $|k(t, x)| \leqslant a(\phi(t)|x|)$, for all $(t, x) \in \mathfrak{R}^{+} \times U$.

\section{Robust Forward Completeness}

In this section, we study the properties of forward complete time-varying systems with locally Lipschitz dynamics. Particularly, we are interested in systems that are "robustly" forward complete with respect to measurable inputs and have an equilibrium point. Next we give the definition of robust forward completeness.

Definition 2.1. We say that (1.1) is Robustly Forward Complete (RFC) if for every $T \geqslant 0, r \geqslant 0$ it holds that $\sup \left\{\left|x\left(t_{0}+h, t_{0}, x_{0} ; d\right)\right| ;\left|x_{0}\right| \leqslant r, t_{0} \in[0, T]\right.$, $\left.h \in[0, T], d(\cdot) \in M_{D}\right\}<+\infty$.

Clearly, the notion of robust forward completeness implies the usual notion of forward completeness, which simply requires that for every initial condition the solution of the system exists for all times greater than the initial time (or equivalently, the solutions of the system do not present finite escape time). However, Proposition 5.1 in [12] shows that every forward complete system (1.1) with dynamics, which are locally Lipschitz with respect to $(t, x)$, uniformly in $d \in D$, is RFC. The following theorem is the main result of this section, which provides Lyapunov-like characterizations of RFC for system (1.1) when the dynamics are continuous with respect to the time. Moreover, it generalizes the Lyapunov-like characterization given in [1].

Theorem 2.2. Suppose that $f \in C^{0}\left(\mathfrak{R}^{+} \times \mathfrak{R}^{n} \times\right.$ $\left.D ; \Re^{n}\right)$. Then the following statements are equivalent:

(i) System (1.1) is RFC.

(ii) There exist functions $\beta_{1}(\cdot), \beta_{2}(\cdot) \in K^{+}, a_{1}(\cdot) \in$ $K_{\infty}, a_{2}(\cdot) \in K_{\infty}$ and a function $W \in C^{\infty}\left(\mathfrak{R}^{+} \times\right.$ $\left.\mathfrak{R}^{n} ; \mathfrak{R}^{+}\right)$such that for all $(t, x, d) \in \mathfrak{R}^{+} \times \mathfrak{R}^{n} \times$ $D$, it holds that

$$
\begin{aligned}
& a_{1}\left(\beta_{1}(t)|x|\right) \leqslant W(t, x) \leqslant a_{2}\left(\beta_{2}(t)|x|\right), \\
& \frac{\partial W}{\partial t}(t, x)+\frac{\partial W}{\partial x}(t, x) f(t, x, d) \leqslant-W(t, x) .
\end{aligned}
$$

(iii) There exist functions $\beta_{1}(\cdot), \beta_{2}(\cdot) \in K^{+}, \beta_{3}(\cdot)$, $\beta_{4}(\cdot), \sigma(\cdot) \in C^{0}\left(\Re^{+}\right)$being non-negative, $a_{1}(\cdot) \in K_{\infty}, a_{2}(\cdot) \in K_{\infty}$ and a function $V \in$ $C^{1}\left(\mathfrak{R}^{+} \times \mathfrak{R}^{n} ; \mathfrak{R}^{+}\right)$such that for all $(t, x, d) \in$ $\mathfrak{R}^{+} \times \mathfrak{R}^{n} \times D$, it holds that

$$
\begin{aligned}
& a_{1}\left(\beta_{1}(t)|x|\right) \leqslant V(t, x) \\
& \leqslant a_{2}\left(\beta_{2}(t)|x|\right)+\beta_{3}(t), \\
& \frac{\partial V}{\partial t}(t, x)+ \frac{\partial V}{\partial x}(t, x) f(t, x, d) \\
& \leqslant \sigma(t) V(t, x)+\beta_{4}(t) .
\end{aligned}
$$

The proof of Theorem 2.2 is based on the following lemma, which provides some elementary characterizations of robustly forward complete systems. It should be emphasized that Lemma 2.3 does not assume continuity of the dynamics with respect to time. We also remind the readers that the notion of non-uniform in time Robust Global Asymptotic Stability (RGAS) was given in [8].

Lemma 2.3. The following statements are equivalent:

(i) System (1.1) is RFC.

(ii) There exist functions $\mu(\cdot) \in K^{+}, a(\cdot) \in K_{\infty}$ and a constant $R \geqslant 0$, such that for every input $d(\cdot) \in$ $M_{D}$ and for every $\left(t_{0}, x_{0}\right) \in \mathfrak{R}^{+} \times \mathfrak{R}^{n}$, the unique 
solution $x(t)$ of (1.1) corresponding to $d(\cdot)$ and initiated from $x_{0}$ at time $t_{0}$ exists for all $t \geqslant t_{0}$ and satisfies:

$$
|x(t)| \leqslant \mu(t)\left(a\left(\left|x_{0}\right|\right)+R\right), \quad \forall t \geqslant t_{0} .
$$

(iii) There exists a function $\beta(\cdot) \in K^{+} \cap C^{\infty}\left(\Re^{+}\right)$, such that the state transformation $x=\beta(t) z$, transforms (1.1) to the following system for which $0 \in$ $\mathfrak{R}^{n}$ is non-uniformly in time RGAS:

$$
\begin{aligned}
& \dot{z}=-\frac{\dot{\beta}(t)}{\beta(t)} z+\frac{1}{\beta(t)} f(t, \beta(t) z, d), \\
& z \in \mathfrak{R}^{n}, \quad t \geqslant 0, \quad d \in D .
\end{aligned}
$$

(iv) System (1.1) is RFC and there exist functions $\mu(\cdot) \in K^{+}, a(\cdot) \in K_{\infty}$, such that for every input $d(\cdot) \in M_{D}$ and for every $\left(t_{0}, x_{0}\right) \in \mathfrak{R}^{+} \times \mathfrak{R}^{n}$, the unique solution $x(t)$ of (1.1) corresponding to $d(\cdot)$ and initiated from $x_{0}$ at time $t_{0}$ satisfies (2.4) with $R=0$.

Proof. Implication (iv) $\Rightarrow$ (i) is obvious, since (2.4) with $R=0$, implies that for every $T \geqslant 0, r \geqslant 0$, it holds that:

$\sup \left\{\left|x\left(t_{0}+h, t_{0}, x_{0} ; d\right)\right| ;\left|x_{0}\right| \leqslant r, t_{0} \in[0, T]\right.$,

$\left.h \in[0, T], d(\cdot) \in M_{D}\right\} \leqslant a(r) \max _{0 \leqslant \tau \leqslant 2 T} \mu(\tau)<+\infty$.

Therefore, we focus on implications (i) $\Rightarrow$ (ii), (ii) $\Rightarrow$ (iii) and (iii) $\Rightarrow$ (iv).

(i) $\Rightarrow$ (ii): We define

$\omega(T, r):=\sup \left\{\left|x\left(t_{0}+h, t_{0}, x_{0} ; d\right)\right| ;\left|x_{0}\right| \leqslant r\right.$,

$\left.t_{0} \in[0, T], h \in[0, T], d(\cdot) \in M_{D}\right\}$.

Notice that by virtue of Definition 2.1, the function $\omega$ : $\left(\mathfrak{R}^{+}\right)^{2} \rightarrow \mathfrak{R}^{+}$is finite valued and for each fixed $t \geqslant 0$ the mappings $\omega(t, \cdot)$ and $\omega(\cdot, t)$ are non-decreasing. Moreover, definition (2.6) implies that for every input $d(\cdot) \in M_{D}$ and for every $\left(t_{0}, x_{0}\right) \in \mathfrak{R}^{+} \times \mathfrak{R}^{n}$, the unique solution $x(t)$ of (1.1) corresponding to $d(\cdot)$ and initiated from $x_{0}$ at time $t_{0}$ satisfies

$|x(t)| \leqslant \omega\left(t,\left|x_{0}\right|\right), \quad \forall t \geqslant t_{0}$.

Since for each fixed $t \geqslant 0$ the mappings $\omega(t, \cdot)$ and $\omega(\cdot, t)$ are non-decreasing, inequality (2.7) implies

$|x(t)| \leqslant \omega(t, t)+\omega\left(\left|x_{0}\right|,\left|x_{0}\right|\right), \quad \forall t \geqslant t_{0}$.
Let $\mu \in K^{+}$be a non-decreasing continuous function that satisfies $1+\omega(s, s) \leqslant \mu(s)$ for all $s \geqslant 0$ and define

$a(s):=s+\mu(s)-\mu(0), \quad R:=1+\mu(0)$

with $a \in K_{\infty}$. It follows from (2.8) that the following inequalities hold for all $t \geqslant t_{0}$ :

$$
\begin{aligned}
|x(t)| & \leqslant \mu(t)+\mu\left(\left|x_{0}\right|\right) \leqslant \mu(t)\left(1+\mu\left(\left|x_{0}\right|\right)\right) \\
& \leqslant \mu(t)\left(R+a\left(\left|x_{0}\right|\right)\right) .
\end{aligned}
$$

Consequently, we conclude that inequality (2.4) holds.

(ii) $\Rightarrow$ (iii): Without loss of generality, we may assume that the function $\mu(\cdot) \in K^{+}$, involved in (2.4), is of class $C^{\infty}$. Consider the following time-varying state transformation for (1.1):

$z:=\frac{1}{\beta(t)} x$,

$\beta(t):=\exp (t) \mu(t)$.

Clearly, we obtain that $z(t)$ satisfies the differential equation (2.5) and moreover, by virtue of (2.4) we have

$$
\begin{gathered}
|z(t)| \leqslant \exp (-t)\left[a\left(\exp \left(t_{0}\right) \mu\left(t_{0}\right)\left|z_{0}\right|\right)+R\right], \\
\forall t \geqslant t_{0}, \quad d(\cdot) \in M_{D} .
\end{gathered}
$$

Estimate (2.12) in conjunction with Proposition 2.5 in [8], implies that $0 \in \mathfrak{R}^{n}$ is non-uniformly in time RGAS for (2.5).

(iii) $\Rightarrow$ (iv): Proposition 2.2 in [8] asserts the existence of functions $\sigma \in K L$ and $\gamma \in K^{+}$such that the solution $z(t)$ of (2.5) satisfies the estimate

$$
\begin{aligned}
& |z(t)| \leqslant \sigma\left(\gamma\left(t_{0}\right)\left|z_{0}\right|, t-t_{0}\right), \quad \forall d(\cdot) \in M_{D} \\
& \quad \text { and } t \geqslant t_{0} .
\end{aligned}
$$

Inequality (2.13) and the fact that $x=\beta(t) z$, for some $\beta \in K^{+}$, implies that

$$
\begin{aligned}
& |x(t)| \leqslant \beta(t) \tilde{a}\left(\frac{\gamma\left(t_{0}\right)}{\beta\left(t_{0}\right)}\left|x_{0}\right|\right), \quad \forall d(\cdot) \in M_{D} \\
& \quad \text { and } t \geqslant t_{0},
\end{aligned}
$$

where $\tilde{a}(s):=\sigma(s, 0)$. Clearly, Fact I implies the existence a function $a(\cdot) \in K_{\infty}$ such that

$$
\tilde{a}(r s) \leqslant a(r) a(s), \quad \forall(r, s) \in\left(\mathfrak{R}^{+}\right)^{2} .
$$

Define

$$
\mu(t):=\beta(t) a\left(\max _{0 \leqslant \tau \leqslant t} \frac{\gamma(\tau)}{\beta(\tau)}\right) .
$$


Inequalities (2.14), (2.15) and definition (2.16) imply (2.4) with $R=0$. The proof is complete

By making use of the equivalence of the statements of Lemma 2.3, we provide the proof of Theorem 2.2.

Proof of Theorem 2.2. (i) $\Rightarrow$ (ii): Suppose that (1.1) is RFC. Then by virtue of statement (iii) of Lemma 2.2 there exists function $\beta(\cdot) \in K^{+} \cap C^{\infty}\left(\Re^{+}\right)$, such that the state transformation $x=\beta(t) z$, transforms (1.1) to system (2.5) for which $0 \in \mathfrak{R}^{n}$ is non-uniformly in time RGAS. Theorem 3.1 in [8] guarantees the existence of functions $\gamma(\cdot) \in K^{+}, a_{1}(\cdot) \in K_{\infty}, a_{2}(\cdot) \in$ $K_{\infty}$ and a function $\tilde{W} \in C^{\infty}\left(\mathfrak{R}^{+} \times \mathfrak{R}^{n} ; \mathfrak{R}^{+}\right)$such that for all $(t, z, d) \in \mathfrak{R}^{+} \times \mathfrak{R}^{n} \times D$, it holds that

$$
\begin{aligned}
& a_{1}(|z|) \leqslant \tilde{W}(t, z) \leqslant a_{2}(\gamma(t)|z|), \\
& \begin{aligned}
& \frac{\partial \tilde{W}}{\partial t}(t, z)+\frac{\partial \tilde{W}}{\partial z}(t, z) \\
& \times {\left[-\frac{\dot{\beta}(t)}{\beta(t)} z+\frac{1}{\beta(t)} f(t, \beta(t) z, d)\right] } \\
& \leqslant-\tilde{W}(t, z) .
\end{aligned}
\end{aligned}
$$

Define

$\beta_{1}(t):=\frac{1}{\beta(t)}, \quad \beta_{2}(t):=\frac{\gamma(t)}{\beta(t)}$,

$W(t, x):=\tilde{W}\left(t, \frac{1}{\beta(t)} x\right)$.

Inequalities $(2.17 \mathrm{a}, \mathrm{b})$ and definitions $(2.18 \mathrm{a}, \mathrm{b})$ imply that inequalities $(2.2 \mathrm{a}, \mathrm{b})$ hold for $W(t, x)$.

(ii) $\Rightarrow$ (iii): This implication is obvious.

(iii) $\Rightarrow$ (i): We evaluate the time derivative of $V(t, x(t))$ along the unique solution $x(t)$ of (1.1) corresponding to $d(\cdot) \in M_{D}$ and initiated from $x_{0}$ at time $t_{0}$ :

$\frac{\mathrm{d}}{\mathrm{d} t} V(t, x(t)) \leqslant \sigma(t) V(t, x(t))+\beta_{4}(t)$,

a.e. for $t \geqslant t_{0}$.

Then by virtue of (2.3a) and (2.19) we have

$$
\begin{aligned}
|x(t)| \leqslant & \frac{1}{\beta_{1}(t)} a_{1}^{-1}\left(\exp \left(\int_{0}^{t} \sigma(s) \mathrm{d} s\right)\right. \\
& \left.\times a_{2}\left(\beta_{2}\left(t_{0}\right)\left|x_{0}\right|\right)+\tilde{\beta}_{4}(t)\right), \\
& \forall t \geqslant t_{0},
\end{aligned}
$$

where $\tilde{\beta}_{4}(t):=\exp \left(\int_{0}^{t} \sigma(s) \mathrm{d} s\right)\left[\int_{0}^{t} \beta_{4}(\tau) \mathrm{d} \tau+\beta_{3}(t)\right]$. It follows from (2.20) that the following inequality is satisfied for all $r, T \geqslant 0$ :

$$
\begin{aligned}
\sup & \left\{\left|x\left(t_{0}+h\right)\right| ;\left|x_{0}\right| \leqslant r, t_{0} \in[0, T],\right. \\
& \left.h \in[0, T], d(\cdot) \in M_{D}\right\} \\
\leqslant & \frac{1}{\min _{t \in[0,2 T]} \beta_{1}(t)} a_{1}^{-1}\left(\exp \left(\int_{0}^{2 T}|\sigma(s)| \mathrm{d} s\right)\right. \\
& \left.\times a_{2}\left(r \max _{t_{0} \in[0, T]} \beta_{2}\left(t_{0}\right)\right)+\max _{t \in[0,2 T]} \tilde{\beta}_{4}(t)\right) \\
< & +\infty .
\end{aligned}
$$

Consequently, by Definition 2.1, we conclude that system (1.1) is RFC. The proof is complete.

Example 2.4. Consider the system

$\dot{x}=c_{1}(t, d) x+c_{2}(t, d) x^{2}+c_{3}(t, d) x^{3}$,

$x \in \mathfrak{R}, \quad t \geqslant 0, \quad d \in D$,

where $D \subset \mathfrak{R}^{m}$ is a compact set, $c_{i}(\cdot) \in C^{0}\left(\mathfrak{R}^{+} \times\right.$ D) $i=1,2,3$ are continuous mappings for which there exists a function $\phi \in K^{+}$such that

$2 \phi(t) c_{3}(t, d) \leqslant-\left|c_{2}(t, d)\right|, \quad \forall(t, d) \in \mathfrak{R}^{+} \times D$.

Next we prove, that this system is RFC. Consider the Lyapunov function

$$
\begin{aligned}
W(t, x):= & \exp \left(-\int_{0}^{t}\left(1+2 \max _{d \in D}\left|c_{1}(\tau, d)\right|\right.\right. \\
& \left.\left.+\phi(\tau) \max _{d \in D}\left|c_{2}(\tau, d)\right|\right) \mathrm{d} \tau\right)|x|^{2} .
\end{aligned}
$$

Clearly, we have for all $(t, x, d) \in \mathfrak{R}^{+} \times \mathfrak{R} \times D$ :

$$
\begin{aligned}
\frac{\partial W}{\partial t}( & t, x)+\frac{\partial W}{\partial x}(t, x)\left(c_{1}(t, d) x\right. \\
& \left.+c_{2}(t, d) x^{2}+c_{3}(t, d) x^{3}\right) \\
= & -\left(1+2 \max _{d \in D}\left|c_{1}(t, d)\right|\right. \\
& \left.+\phi(t) \max _{d \in D}\left|c_{2}(t, d)\right|\right) W(t, x) \\
& +\left(2 c_{1}(t, d) x^{2}+2 c_{2}(t, d) x^{3}+2 c_{3}(t, d) x^{4}\right) \\
& \times \exp \left(-\int_{0}^{t}\left(1+2 \max _{d \in D}\left|c_{1}(\tau, d)\right|\right.\right. \\
& \left.\left.+\phi(\tau) \max _{d \in D}\left|c_{2}(\tau, d)\right|\right) \mathrm{d} \tau\right) .
\end{aligned}
$$


It follows from inequality (2.23) in conjunction with the Young inequality

$2 c_{2}(t, d) x^{3} \leqslant \phi(t)\left|c_{2}(t, d)\right| x^{2}+\frac{\left|c_{2}(t, d)\right|}{\phi(t)} x^{4}$,

that the following inequality holds:

$$
\begin{aligned}
& \frac{\partial W}{\partial t}(t, x)+\frac{\partial W}{\partial x}(t, x)\left(c_{1}(t, d) x+c_{2}(t, d) x^{2}\right. \\
& \left.\quad+c_{3}(t, d) x^{3}\right) \leqslant-W(t, x), \\
& \quad \forall(t, x, d) \in \mathfrak{R}^{+} \times \mathfrak{R} \times D .
\end{aligned}
$$

Moreover, inequality (2.2a) holds with $a_{1}(s)=$ $a_{2}(s):=s^{2}$,

$$
\begin{aligned}
\beta_{1}(t):= & \exp \left(-\frac{1}{2} \int_{0}^{t}\left(1+2 \max _{d \in D}\left|c_{1}(\tau, d)\right|\right.\right. \\
& \left.\left.+\phi(\tau) \max _{d \in D}\left|c_{2}(\tau, d)\right|\right) \mathrm{d} \tau\right), \\
\beta_{2}(t) \equiv & 1 .
\end{aligned}
$$

Thus system (2.22) is RFC.

\section{Robust Global Asymptotic Output Stability}

In this section we introduce the notion of (nonuniform in time) Robust Global Asymptotic Output Stability (RGAOS) as a generalization of the notion of robust output stability, given in $[14,15]$. Let us denote by $H\left(t, x\left(t, t_{0}, x_{0} ; d\right)\right)=Y(t)$ the value of output (1.2) for the unique solution of (1.1) at time $t$ that corresponds to input $d \in M_{D}$ with initial condition $x\left(t_{0}\right)=x_{0}$.

Definition 3.1. Consider system (1.1) with output (1.2). Suppose that (1.1) is RFC. We say that system (1.1) with output (1.2) is (non-uniformly in time) Robust Global Asymptotic Output Stability (RGAOS) if it satisfies the following properties:

P1 (Output Stability). For every $\varepsilon>0, T \geqslant 0$, it holds that

$$
\begin{aligned}
& \sup \left\{|Y(t)| ; t \geqslant t_{0},\left|x_{0}\right| \leqslant \varepsilon, t_{0} \in[0, T],\right. \\
& \left.d(\cdot) \in M_{D}\right\}<+\infty
\end{aligned}
$$

and there exists a $\delta:=\delta(\varepsilon, T)>0$ such that

$$
\begin{aligned}
& \left|x_{0}\right| \leqslant \delta, t_{0} \in[0, T] \Rightarrow|Y(t)| \leqslant \varepsilon, \quad \forall t \geqslant t_{0}, \\
& \quad \forall d(\cdot) \in M_{D} .
\end{aligned}
$$

P2 (Uniform output attractivity on compact sets of initial data). For every $\varepsilon>0, T \geqslant 0$ and $R \geqslant 0$, there exists a $\tau:=\tau(\varepsilon, T, R) \geqslant 0$, such that

$\left|x_{0}\right| \leqslant R, \quad t_{0} \in[0, T] \Rightarrow|Y(t)| \leqslant \varepsilon$,

$\forall t \geqslant t_{0}+\tau, \quad \forall d(\cdot) \in M_{D}$.

We say that system (1.1) with output (1.2) is (nonuniformly in time) strongly RGAOS if it is nonuniformly in time RGAOS and the set $H^{-1}(t, 0):=$ $\left\{x \in \mathfrak{R}^{n} ; H(t, x)=0\right\}$ is positively invariant.

Notice that property $\mathrm{P} 2$ is stronger than the usual output attractivity property $\left(\lim _{t \rightarrow+\infty} Y(t)=0\right)$ even in the autonomous case. It is clear that the notion of non-uniform in time RGAOS coincides with the notion of non-uniform in time RGAS given in [8], when $H(t, x)=x$. Moreover, if there exists $a \in K_{\infty}$ such that $|x| \leqslant a(|H(t, x)|)$ for all $(t, x) \in \mathfrak{R}^{+} \times \mathfrak{R}^{n}$, then non-uniform in time RGAOS implies non-uniform in time RGAS. The following theorem is the main result of this section and provides Lyapunov-like characterizations of the notion of non-uniform in time RGAOS when the dynamics are continuous with respect to the time.

Theorem 3.2. Suppose that $H \in C^{0}\left(\mathfrak{R}^{+} \times \mathfrak{R}^{n} ; \mathfrak{R}^{k}\right)$ and $f \in C^{0}\left(\mathfrak{R}^{+} \times \mathfrak{R}^{n} \times D ; \mathfrak{R}^{n}\right)$. Then the following statements are equivalent:

(i) System (1.1) with output (1.2) is non-uniformly in time RGAOS.

(ii) There exist functions $\beta_{1}(\cdot), \beta_{2}(\cdot) \in K^{+}, a_{1}(\cdot) \in$ $K_{\infty}, a_{2}(\cdot) \in K_{\infty}$ and a function $U \in C^{\infty}\left(\mathfrak{R}^{+} \times\right.$ $\left.\mathfrak{R}^{n} ; \mathfrak{R}^{+}\right)$such that

$$
\begin{gathered}
a_{1}\left(\left|\left(\beta_{1}(t) x, H(t, x)\right)\right|\right) \leqslant U(t, x) \leqslant a_{2}\left(\beta_{2}(t)|x|\right), \\
\forall(t, x) \in \mathfrak{R}^{+} \times \mathfrak{R}^{n}, \\
\frac{\partial U}{\partial t}(t, x)+\frac{\partial U}{\partial x}(t, x) f(t, x, d) \leqslant-U(t, x), \\
\forall(t, x, d) \in \mathfrak{R}^{+} \times \mathfrak{R}^{n} \times D .
\end{gathered}
$$

(iii) There exist functions $\beta_{1}(\cdot), \beta_{2}(\cdot) \in K^{+}, \phi(\cdot) \in \mathrm{E}$ (see Notations for the definition of class $\mathrm{E}, a_{1}(\cdot)$, $a_{2}(\cdot) \in K_{\infty}, \rho(\cdot) \in C^{0}\left(\mathfrak{R}^{+} ; \mathfrak{R}^{+}\right)$being positive definite and $V \in C^{1}\left(\mathfrak{R}^{+} \times \mathfrak{R}^{n} ; \mathfrak{R}^{+}\right)$such that

$$
\begin{gathered}
a_{1}\left(\left|\left(\beta_{1}(t) x, H(t, x)\right)\right|\right) \leqslant V(t, x) \leqslant a_{2}\left(\beta_{2}(t)|x|\right), \\
\forall(t, x) \in \mathfrak{R}^{+} \times \mathfrak{R}^{n},
\end{gathered}
$$




$$
\begin{gathered}
\frac{\partial V}{\partial t}(t, x)+\frac{\partial V}{\partial x}(t, x) f(t, x, d) \\
\leqslant-\rho(V(t, x))+\phi(t), \\
\forall(t, x, d) \in \mathfrak{R}^{+} \times \mathfrak{R}^{n} \times D .
\end{gathered}
$$

The proof of Theorem 3.2 is based on the following technical lemmas, which provide estimates of the trajectories in terms of $K L$ functions. It should be emphasized that the following lemmas do not assume continuity of the dynamics with respect to time. The proof of the following lemma is identical with the proof of Proposition 2.2 in [8] and is omitted.

Lemma 3.3. Suppose that system (1.1) is RFC. Then system (1.1) with output (1.2) is non-uniformly in time RGAOS if and only if there exist functions $\sigma \in K L$ and $\beta \in K^{+}$such that for every $t_{0} \geqslant 0$ and $x_{0} \in \mathfrak{R}^{n}$ the following estimate holds:

$$
\begin{array}{r}
|Y(t)| \leqslant \sigma\left(\beta\left(t_{0}\right)\left|x_{0}\right|, t-t_{0}\right), \\
\forall d(\cdot) \in M_{D} \text { and } t \geqslant t_{0} .
\end{array}
$$

An immediate consequence of the equivalence of statements (i) and (iv) of Lemma 2.3 and the result of Lemma 3.3 is the following preliminary' characterization of the notion of non-uniform in time RGAOS in terms of $K L$ functions.

Lemma 3.4. The following statements are equivalent:

(i) System (1.1) with output (1.2) is non-uniformly in time RGAOS.

(ii) There exist functions $\sigma \in K L$ and $\beta, \mu \in K^{+}$such that for every $t_{0} \geqslant 0$ and $x_{0} \in \mathfrak{R}^{n}$ the following estimate holds:

$$
\begin{gathered}
\mu(t)|x(t)|+|Y(t)| \leqslant \sigma\left(\beta\left(t_{0}\right)\left|x_{0}\right|, t-t_{0}\right), \\
\forall d(\cdot) \in M_{D} \text { and } t \geqslant t_{0} .
\end{gathered}
$$

Proof. Since the proof of (ii) $\Rightarrow$ (i) is an immediate consequence of the properties of the $K L$ functions, we prove only implication (i) $\Rightarrow$ (ii). Suppose that system (1.1) with output (1.2) is non-uniformly in time RGAOS. Then, by virtue of Lemma 3.3, there exist functions $\tilde{\sigma} \in K L$ and $\tilde{\beta} \in K^{+}$such that for every $t_{0} \geqslant 0$ and $x_{0} \in \mathfrak{R}^{n}$ the following estimate holds:

$$
\begin{array}{r}
|Y(t)| \leqslant \tilde{\sigma}\left(\tilde{\beta}\left(t_{0}\right)\left|x_{0}\right|, t-t_{0}\right), \\
\forall d(\cdot) \in M_{D} \text { and } t \geqslant t_{0} .
\end{array}
$$

Since system (1.1) is RFC, by virtue of the equivalence of statements (i) and (iv) of Lemma 2.3, there exist functions $\tilde{\mu} \in K^{+}, a \in K_{\infty}$, such that for every $\left(t_{0}, x_{0}\right) \in \mathfrak{R}^{+} \times \mathfrak{R}^{n}$, the following estimate holds:

$\frac{1}{\tilde{\mu}(t)}|x(t)| \leqslant a\left(\left|x_{0}\right|\right), \quad \forall d(\cdot) \in M_{D}$ and $t \geqslant t_{0}$,

which directly implies the following estimate:

$$
\begin{gathered}
\mu(t)|x(t)| \leqslant \exp \left(-\left(t-t_{0}\right)\right) a\left(\left|x_{0}\right|\right), \\
\forall d(\cdot) \in M_{D} \text { and } t \geqslant t_{0},
\end{gathered}
$$

where $\mu(t):=\exp (-t) / \tilde{\mu}(t)$. Inequalities (3.5) and (3.6) imply estimate (3.4) with $\beta(t):=1+\tilde{\beta}(t)$ and $\sigma(s, t):=\tilde{\sigma}(s, t)+\exp (-t) a(s)$. The proof is complete.

The following lemma shows that if property P2 of Definition 3.1 is satisfied, then property P1 of Definition 3.1 automatically holds. Thus in order to establish non-uniform in time RGAOS, we only need to establish property P2 of Definition 3.1 and RFC.

Lemma 3.5. (Uniform output attractivity on compact sets of initial data and RFC Implies Output Stability). Consider system (1.1) with output (1.2), where $H$ : $\mathfrak{R}^{+} \times \mathfrak{R}^{n} \rightarrow \mathfrak{R}^{k}$ is a $C^{0}$ function with $H(t, 0)=0$ for all $t \geqslant 0$. Suppose that (1.1) is RFC. Then system (1.1), with output (1.2) is (non-uniformly in time) RGAOS if Property P2 of Definition 3.1 holds.

Proof. Suppose that system (1.1) is RFC and that property P2 (uniform output attractivity on compact sets of initial data) of Definition 3.1 holds. Then it suffices to show that property P1 (Output Stability) of Definition 3.1 also holds. Since (1.1) is RFC, it follows by Lemma 2.2 that there exist functions $\mu(\cdot) \in$ $K^{+}, a(\cdot) \in K_{\infty}$, such that for every input $d(\cdot) \in M_{D}$ and for every $\left(t_{0}, x_{0}\right) \in \mathfrak{R}^{+} \times \mathfrak{R}^{n}$, the unique solution $x(t)$ of (1.1) corresponding to $d(\cdot)$ and initiated from $x_{0}$ at time $t_{0}$ exists for all $t \geqslant t_{0}$ and satisfies

$|x(t)| \leqslant \mu(t) a\left(\left|x_{0}\right|\right), \quad \forall t \geqslant t_{0}$.

We next show that property P1 (Output Stability) of Definition 3.1 holds. Let arbitrary $\varepsilon>0$ and $T \geqslant 0$. By virtue of property P2 there exists a $\tau:=\tau(\varepsilon, T) \geqslant 0$, such that

$$
\begin{gathered}
\left|x_{0}\right| \leqslant \varepsilon, \quad t_{0} \in[0, T] \Rightarrow|Y(t)| \leqslant \varepsilon, \\
\forall t \geqslant t_{0}+\tau, \quad \forall d(\cdot) \in M_{D} .
\end{gathered}
$$


Since $H(\cdot)$ is continuous with $H(t, 0)=0$ for all $t \geqslant 0$, by virtue of Fact II, there exist functions $\zeta \in K_{\infty}$ and $\gamma \in K^{+}$such that

$|H(t, x)| \leqslant \zeta(\gamma(t)|x|), \quad \forall(t, x) \in \mathfrak{R}^{+} \times \mathfrak{R}^{n}$.

By virtue of inequalities (3.7), (3.9) and property (3.8) it holds that

$$
\begin{aligned}
& \sup \left\{|Y(t)| ; t \geqslant t_{0},\left|x_{0}\right| \leqslant \varepsilon, t_{0} \in[0, T], d(\cdot) \in M_{D}\right\} \\
& \leqslant \max (\varepsilon, \zeta(a(\varepsilon) M(\varepsilon, T))), \\
& M(\varepsilon, T):=\max _{0 \leqslant s \leqslant T+\tau(\varepsilon, T)} \gamma(s) \mu(s)>0 .
\end{aligned}
$$

Finally, we define

$\delta(\varepsilon, T):=\min \left(\varepsilon, a^{-1}\left(\frac{\zeta^{-1}(\varepsilon)}{M(\varepsilon, T)}\right)\right)>0$.

Clearly, by virtue of inequalities (3.7) and (3.9) and definitions (3.10b), (3.11), we obtain that

$$
\begin{gathered}
\left|x_{0}\right| \leqslant \delta(\varepsilon, T), \quad t_{0} \in[0, T] \Rightarrow|Y(t)| \leqslant \varepsilon, \\
\forall t \in\left[t_{0}, t_{0}+\tau(\varepsilon, T)\right], \quad \forall d(\cdot) \in M_{D} .
\end{gathered}
$$

The latter property, in conjunction with properties (3.8), (3.10a) and definition (3.11), implies that property P1 of Definition 3.1 holds. The proof is complete.

Obviously, if zero for system (1.1) is non-uniformly in time RGAS and the output function is timeinvariant, i.e. $H(t, x) \equiv H(x)$ then system (1.1) with output (1.2) is non-uniformly in time RGAOS. However, this conclusion does not hold if the output function actually depends on time, as the following example shows.

Example 3.6. Consider the system:

$\dot{x}=-(2+d) x$,

$x \in \mathfrak{R}, \quad d \in[-1,1]$.

It is clear that zero is (uniformly in time) RGAS for system (3.12). Clearly, the solution of (3.12) with initial condition $x\left(t_{0}\right)=x_{0}$ satisfies

$\exp \left(-3\left(t-t_{0}\right)\right)\left|x_{0}\right| \leqslant|x(t)| \leqslant \exp \left(-\left(t-t_{0}\right)\right)\left|x_{0}\right|$, $\forall d(\cdot) \in M_{[-1,1]}, \quad t \geqslant t_{0}$.

However, if we define the output

$Y=H(t, x)=\exp (4 t) x$, then obviously, system (3.12) with output (3.13) is not RGAOS.

The following proposition provides a Lyapunov-like characterization for a time-varying system, which is non-uniformly in time RGAOS and for which zero is non-uniformly in time RGAS.

Proposition 3.7. Suppose that $H \in C^{0}\left(\Re^{+} \times\right.$ $\left.\mathfrak{R}^{n} ; \mathfrak{R}^{k}\right)$ and $f \in C^{0}\left(\mathfrak{R}^{+} \times \mathfrak{R}^{n} \times D ; \mathfrak{R}^{n}\right)$. Then system (1.1) with output (1.2) is non-uniformly in time RGAOS and zero is non-uniformly in time RGAS for (1.1) if and only if there exist functions $U(\cdot) \in C^{\infty}\left(\mathfrak{R}^{+} \times \mathfrak{R}^{n} ; \mathfrak{R}^{+}\right), a_{1}(\cdot), a_{2}(\cdot) \in K_{\infty}$ and $\beta(\cdot) \in K^{+}$such that

$$
\begin{gathered}
a_{1}(|(x, H(t, x))|) \leqslant U(t, x) \leqslant a_{2}(\beta(t)|x|), \\
\forall(t, x) \in \mathfrak{R}^{+} \times \mathfrak{R}^{n},
\end{gathered}
$$

$$
\begin{gathered}
\frac{\partial U}{\partial t}(t, x)+\frac{\partial U}{\partial x}(t, x) f(t, x, d) \leqslant-U(t, x), \\
\forall(t, x, d) \in \mathfrak{R}^{+} \times \mathfrak{R}^{n} \times D .
\end{gathered}
$$

Proof. If the dynamics $f \in C^{0}\left(\mathfrak{R}^{+} \times \mathfrak{R}^{n} \times D ; \mathfrak{R}^{n}\right)$ were locally Lipschitz in both variables $(t, x)$ then Proposition 3.7 would follow directly from the equivalence of statements (i) and (ii) of Lemma 3.4 and an immediate application of the converse Lyapunov Theorem in [16]. However, we have only assumed continuity with respect to time and in order to prove Proposition 3.5 we have to follow a different procedure. Since $H(\cdot)$ is continuous with $H(t, 0)=0$ for all $t \geqslant 0$, by virtue of Fact II, there exists $\tilde{\zeta} \in K_{\infty}$ and $\kappa \in K^{+}$such that

$|H(t, x)| \leqslant \tilde{\zeta}(\kappa(t)|x|), \quad$ for all $(t, x) \in \mathfrak{R}^{+} \times \mathfrak{R}^{n}$,

which implies that

$$
\begin{aligned}
& |H(t, x)| \leqslant \tilde{\zeta}\left(|x|^{1 / 2}\right), \text { for all } t \geqslant 0 \\
& \text { and }|x| \leqslant \frac{1}{\kappa^{2}(t)} .
\end{aligned}
$$

Consequently, there exists a function $\tilde{H} \in C^{\infty}\left(\mathfrak{R}^{+} \times\right.$ $\mathfrak{R}^{n} ; \mathfrak{R}^{k}$ ) such that

$$
|\tilde{H}(t, x)-H(t, x)| \leqslant 2 \tilde{\zeta}\left(|x|^{1 / 2}\right),
$$

for all $(t, x) \in \mathfrak{R}^{+} \times \mathfrak{R}^{n}$, 
$\tilde{H}(t, x)=0, \quad$ for all $t \geqslant 0$

and $|x| \leqslant \frac{1}{\kappa^{2}(t)}$.

By virtue of Lemma 3.3, Proposition 2.2 in [8] and the facts that system (1.1) with output (1.2) is nonuniformly in time RGAOS and zero is non-uniformly in time RGAS for (1.1), there exist functions $\bar{\sigma} \in K L$ and $\tilde{\beta} \in K^{+}$such that

$$
\begin{gathered}
|x(t)|+|H(t, x(t))| \leqslant \bar{\sigma}\left(\tilde{\beta}\left(t_{0}\right)\left|x_{0}\right|, t-t_{0}\right), \\
\forall t \geqslant t_{0}, d(\cdot) \in M_{D}, \quad\left(t_{0}, x_{0}\right) \in \mathfrak{R}^{+} \times \mathfrak{R}^{n} .
\end{gathered}
$$

Inequalities (3.15a) and (3.16) guarantee the existence of a function $\sigma \in K L$ such that

$$
\begin{gathered}
|x(t)|+|\tilde{H}(t, x(t))| \leqslant \sigma\left(\tilde{\beta}\left(t_{0}\right)\left|x_{0}\right|, t-t_{0}\right), \\
\forall t \geqslant t_{0}, \quad d(\cdot) \in M_{D}, \quad\left(t_{0}, x_{0}\right) \in \mathfrak{R}^{+} \times \mathfrak{R}^{n} .
\end{gathered}
$$

Therefore, using the equivalence of statements (i) and (ii) of Lemma 3.4, system (1.1) with output $Y=\tilde{H}(t, x)$ is non-uniformly in time RGAOS and zero is non-uniformly in time RGAS for (1.1). Next, we consider the system

$\dot{x}=f(t, x, d)$,

$\dot{w}=-w+\tilde{H}(t, x)+\frac{\partial \tilde{H}}{\partial t}(t, x)+\frac{\partial \tilde{H}}{\partial x}(t, x) f(t, x, d)$,

$x \in \mathfrak{R}^{n}, w \in \mathfrak{R}^{k}, t \geqslant 0, d \in D$.

We claim that zero for the above system is nonuniformly in time RGAS. Notice that the solution $(x(t), w(t))$ of (3.18) initiated from $\left(x_{0}, w_{0}\right) \in$ $\mathfrak{R}^{n} \times \mathfrak{R}^{k}$ at time $t_{0} \leqslant 0$ and corresponding to $d(\cdot) \in$ $M_{D}$ satisfies

$$
\begin{aligned}
w(t)= & \tilde{H}(t, x(t))+\exp \left(-\left(t-t_{0}\right)\right) \\
& \times\left(w_{0}-\tilde{H}\left(t_{0}, x_{0}\right)\right), \quad \forall t \geqslant t_{0} .
\end{aligned}
$$

Moreover the component $x(t)$ of the solution $(x(t), w(t))$ of (3.18) initiated from $\left(x_{0}, w_{0}\right) \in$ $\mathfrak{R}^{n} \times \mathfrak{R}^{k}$ at time $t_{0} \geqslant 0$ and corresponding to $d(\cdot) \in$ $M_{D}$ coincides with the solution $x(t)$ of (1.1) initiated from $x_{0} \in \mathfrak{R}^{n}$ at time $t_{0} \geqslant 0$ and corresponding to the same $d(\cdot) \in M_{D}$. Since $\tilde{H}(\cdot)$ is continuous with $\tilde{H}(t, 0)=0$ for all $t \geqslant 0$, by virtue of Fact II, there exist functions $\zeta \in K_{\infty}$ and $\gamma \in K^{+}$such that

$$
|\tilde{H}(t, x)| \leqslant \zeta(\gamma(t)|x|), \quad \forall(t, x) \in \mathfrak{R}^{+} \times \mathfrak{R}^{n} .
$$

Clearly, inequalities (3.17), and (3.20), in conjunction with (3.19) imply that

$$
\begin{aligned}
& |(x(t), w(t))| \leqslant \tilde{\sigma}\left(\tilde{\gamma}\left(t_{0}\right)\left|\left(x_{0}, w_{0}\right)\right|, t-t_{0}\right), \\
& \quad \forall t \geqslant t_{0}, \quad d(\cdot) \in M_{D}, \\
& \quad\left(t_{0}, x_{0}, w_{0}\right) \in \mathfrak{R}^{+} \times \mathfrak{R}^{n} \times \mathfrak{R}^{k},
\end{aligned}
$$

where

$\tilde{\gamma}(t):=1+\tilde{\beta}(t)+\gamma(t)$,

$\tilde{\sigma}(s, t):=2 \sigma(s, t)+(s+\zeta(s)) \exp (-t)$.

Inequality (3.21a) implies that zero is non-uniformly in time RGAS for (3.18). Moreover, by virtue of Theorem 3.1 in [8], there exists a function $V(\cdot) \in$ $C^{\infty}\left(\mathfrak{R}^{+} \times \mathfrak{R}^{n} \times \mathfrak{R}^{k} ; \mathfrak{R}^{+}\right)$and functions $\tilde{a}_{1}(\cdot), \tilde{a}_{2}(\cdot) \in$ $K_{\infty}, \beta_{2}(\cdot) \in K^{+}$such that

$$
\begin{aligned}
& \left.\tilde{a}_{1}(\mid x, w) \mid\right) \leqslant V(t, x, w) \leqslant \tilde{a}_{2}\left(\beta_{2}(t)|(x, w)|\right), \\
& \forall(t, x, w) \in \mathfrak{R}^{+} \times \mathfrak{R}^{n} \times \mathfrak{R}^{k}, \\
& \frac{\partial V}{\partial t}(t, x, w)+\frac{\partial V}{\partial x}(t, x, w) f(t, x, d)+\frac{\partial V}{\partial w}(t, x, w) \\
& \quad \times\left(\frac{\partial \tilde{H}}{\partial t}(t, x)+\frac{\partial \tilde{H}}{\partial x}(t, x) f(t, x, d)-w+\tilde{H}(t, x)\right) \\
& \leqslant-V(t, x, w) \\
& \quad \forall(t, x, w, d) \in \mathfrak{R}^{+} \times \mathfrak{R}^{n} \times \mathfrak{R}^{k} \times D .
\end{aligned}
$$

Finally, we define

$U(t, x):=V(t, x, \tilde{H}(t, x))$.

Inequality (3.14b) is an immediate consequence of (3.22b) and definition (3.23). Moreover, by virtue of Fact II, there exist functions $a_{2} \in K_{\infty}$ and $\beta \in K^{+}$ such that

$$
\begin{gathered}
\tilde{a}_{2}\left(\beta_{2}(t) s+\beta_{2}(t) \zeta(\gamma(t) s)\right) \leqslant a_{2}(\beta(t) s), \\
\forall(t, s) \in \mathfrak{R}^{+} \times \mathfrak{R}^{+} .
\end{gathered}
$$

The right-hand-side inequality of (3.14a) is an immediate consequence of inequalities (3.20), (3.22a), (3.24) and definition (3.23). Finally, the left-hand-side inequality (3.14a) follows from (3.15a) and the inequalities

$$
\begin{aligned}
& \tilde{a}_{1}(|(x, \tilde{H}(t, x))|) \geqslant \frac{1}{2} \tilde{a}_{1}(|x|)+\frac{1}{2} \tilde{a}_{1}(|\tilde{H}(t, x)|), \\
& \delta(2|\tilde{H}(t, x)|)+\delta\left(4 \tilde{\zeta}\left(|x|^{1 / 2}\right)\right) \geqslant \delta(|H(t, x)|), \\
& \delta(|H(t, x)|)+\delta(|x|) \geqslant \delta\left(\frac{1}{2}|(x, H(t, x))|\right),
\end{aligned}
$$


where $\delta(s):=\frac{1}{4} \min \left(\tilde{a}_{1}(s / 2), \tilde{a}_{1}\left(\left(\tilde{\zeta}^{-1}(s / 4)\right)^{2}\right)\right)$ and $a_{1}(s):=\delta(s / 2)$ are functions of class $K_{\infty}$.

The converse statement is immediate, if we evaluate the time derivative of $U(t, x(t))$ along the unique solution $x(t)$ of (1.1) corresponding to $d(\cdot) \in M_{D}$ and initiated from $x_{0}$ at time $t_{0}$ we obtain

$\frac{\mathrm{d}}{\mathrm{d} t} U(t, x(t)) \leqslant-U(t, x(t)), \quad$ a.e. for $t \geqslant t_{0}$.

The latter differential inequality implies $U(t, x(t))$ $\leqslant \exp \left(-\left(t-t_{0}\right)\right) U\left(t_{0}, x\left(t_{0}\right)\right)$ for all $t \geqslant t_{0}$ and this in conjunction with (3.14a) guarantees that system (1.1) with output (1.2) is non-uniformly in time RGAOS and zero is non-uniformly in time RGAS for (1.1). The proof is complete.

We next provide the proof of Theorem 3.2.

Proof of Theorem 3.2. (i) $\Rightarrow$ (ii): Since system (1.1) is RFC, by virtue of statement (iii) of Lemma 2.3, it follows that there exists a function $\beta(\cdot) \in K^{+} \cap$ $C^{\infty}\left(\Re^{+}\right)$, such that the state transformation $x=\beta(t) z$, transforms (1.1) to system (2.5) for which $0 \in \mathfrak{R}^{n}$ is non-uniformly in time RGAS. Consider system (2.5) with output

$\tilde{Y}=H(t, \beta(t) z)$.

Since system (1.1) with output (1.2) is non-uniformly in time RGAOS, it follows by Lemma 3.3 that there exist functions $\sigma \in K L$ and $\tilde{\beta} \in K^{+}$such that for every $t_{0} \geqslant 0$ and $x_{0} \in \mathfrak{R}^{n}$ such that

$$
\begin{gathered}
|Y(t)| \leqslant \sigma\left(\tilde{\beta}\left(t_{0}\right)\left|x_{0}\right|, t-t_{0}\right), \\
\forall d(\cdot) \in M_{D} \text { and } t \geqslant t_{0} .
\end{gathered}
$$

The latter inequality implies the following estimate for the output of system (2.5) with output (3.25):

$$
\begin{array}{r}
|\tilde{Y}(t)| \leqslant \sigma\left(\frac{\tilde{\beta}\left(t_{0}\right)}{\beta\left(t_{0}\right)}\left|z_{0}\right|, t-t_{0}\right), \\
\forall d(\cdot) \in M_{D} \text { and } t \geqslant t_{0} .
\end{array}
$$

It follows that system (2.5) with output (3.25) is nonuniformly in time RGAOS and zero is non-uniformly in time RGAS for (2.5). The desired implication is an immediate consequence of Proposition 3.7.

(ii) $\Rightarrow$ (iii): This implication is obvious.

(iii) $\Rightarrow$ (i): Clearly, by virtue of statement (iii) of Theorem 2.2 it follows that system (1.1) is RFC. By virtue of Lemma 3.5, it suffices to show that property P2 of Definition 3.1 holds for system (1.1) with output (1.2). We evaluate the time derivative of $V(t, x(t))$ along the unique solution $x(t)$ of (1.1) corresponding to $d(\cdot) \in M_{D}$ and initiated from $x_{0}$ at time $t_{0}$ :

$$
\begin{aligned}
\frac{\mathrm{d}}{\mathrm{d} t} V(t, x(t)) \leqslant & -\rho(V(t, x(t))), \\
& +\phi(t) \quad \text { a.e. for } t \geqslant t_{0} .
\end{aligned}
$$

Then by virtue of Lemma 3.2 in [8], there exist a function $\sigma \in K L$ and a constant $M \geqslant 0$ such that

$$
\begin{gathered}
V(t, x(t)) \leqslant \sigma\left(V\left(t_{0}, x_{0}\right)+M, t-t_{0}\right), \\
\forall t \geqslant t_{0} \text { and } d(\cdot) \in M_{D} .
\end{gathered}
$$

Inequality (3.29) in conjunction with (3.2a) implies that property P2 of Definition 3.1 holds for system (1.1) with output (1.2). The proof is complete.

Finally, we end this section by providing a characterization of non-uniform in time strong RGAOS. Its proof is trivial and is omitted.

Corollary 3.8. System (1.1) with output (1.2) and $H \in C^{1}\left(\mathfrak{R}^{+} \times \mathfrak{R}^{n} ; \mathfrak{R}^{k}\right), f \in C^{0}\left(\mathfrak{R}^{+} \times \mathfrak{R}^{n} \times D ; \mathfrak{R}^{n}\right)$ is non-uniformly in time strongly RGAOS if and only if it is non-uniformly in time RGAOS and moreover it holds that

$$
\begin{aligned}
& \frac{\partial H}{\partial t}(t, x)+\frac{\partial H}{\partial x}(t, x) f(t, x, d)=0, \\
& \forall(t, d) \in \mathfrak{R}^{+} \times D, \quad x \in H^{-1}(t, 0),
\end{aligned}
$$

where $H^{-1}(t, 0):=\left\{x \in \mathfrak{R}^{n} ; H(t, x)=0\right\}$.

\section{Sufficient conditions for non-uniform in time RGAOS}

The following sufficient conditions for non-uniform in time RGAOS will be used in forthcoming papers concerning RGAOS.

Proposition 4.1. Consider the system

$\dot{x}=f(t, \theta, x, v)$,

$Y=H(t, x)$,

$x \in \mathfrak{R}^{n}, \quad v \in \mathfrak{R}^{m}, \quad \theta \in \Omega, \quad t \geqslant 0$,

where $\Omega \subset \mathfrak{R}^{l}$ is a compact set, $H: \mathfrak{R}^{+} \times \mathfrak{R}^{n} \rightarrow \mathfrak{R}^{k}$ is a $C^{0}$ function with $H(t, 0)=0$ for all $t \geqslant 0$ and 
the dynamics $f$ are $C^{0}$ on $\mathfrak{R}^{+} \times \Omega \times \mathfrak{R}^{n} \times \mathfrak{R}^{m}$ and locally Lipschitz with respect to $(x, v)$, uniformly in $\theta \in \Omega$, with $f(t, \theta, 0,0)=0$. Suppose that there exist functions $\sigma \in K L, a, \rho \in K_{\infty}, \phi, \beta, \mu \in K^{+}$, such that the unique solution of (4.1) initiated from $x_{0} \in \mathfrak{R}^{n}$ at time $t_{0} \geqslant 0$ and corresponding to input $(\theta(\cdot), v(\cdot)) \in$ $M_{\Omega} \times \mathrm{L}_{\mathrm{loc}}^{\infty}\left(\left[t_{0},+\infty\right)\right)$, satisfies the estimates

$$
\begin{aligned}
|Y(t)| & \leqslant \sigma\left(\beta\left(t_{0}\right)\left|x_{0}\right|, t-t_{0}\right)+\sup _{t_{0} \leqslant \tau \leqslant t} \rho(\phi(\tau)|v(\tau)|), \\
\forall t & \geqslant t_{0}, \\
|x(t)| & \leqslant \mu(t)\left(a\left(\beta\left(t_{0}\right)\left|x_{0}\right|\right)+\sup _{t_{0} \leqslant \tau \leqslant t} \rho(\phi(\tau)|v(\tau)|)\right), \\
\forall t & \geqslant t_{0} .
\end{aligned}
$$

Then there exists a $C^{0}$ function $\gamma(t, s): \mathfrak{R}^{+} \times \mathfrak{R}^{+} \rightarrow$ $\mathfrak{R}^{+}$, which is locally Lipschitz with respect to $s$, with $\gamma(t, \cdot) \in K_{\infty}$ for each $t \geqslant 0$, such that the following system is non-uniformly in time RGAOS with input $(\theta, d) \in D:=\Omega \times B:$

$\dot{x}=f(t, \theta, x, \mathrm{~d} \gamma(t,|x|))$,

$Y=H(t, x)$,

where $B$ denotes the closed unit ball in $\mathfrak{R}^{m}$.

Proof. Let $\eta \in K_{\infty}$ be a locally Lipschitz function that satisfies $\eta(s) \leqslant \rho^{-1}(s)$ for all $s \geqslant 0$. Define

$\gamma(t, s):=\frac{1}{\phi(t)} \eta\left(\frac{\exp (-t) s}{2(1+\mu(t))}\right)$.

Clearly, for every $x_{0} \in \mathfrak{R}^{n}, t_{0} \geqslant 0$ and $(\theta(\cdot), d(\cdot)) \in$ $M_{D}$, there exists $t_{1}>t_{0}$ such that the solution of (4.3) initiated from $x_{0} \in \Re^{n}$ at time $t_{0} \geqslant 0$ and corresponding to input $(\theta(\cdot), d(\cdot)) \in M_{D}$ exists on $\left[t_{0}, t_{1}\right)$. Consequently, by virtue of (4.2b) and (4.4), it satisfies

$$
\begin{aligned}
& \frac{|x(t)|}{\mu(t)} \leqslant a\left(\beta\left(t_{0}\right)\left|x_{0}\right|\right) \\
&+ \frac{1}{2} \sup _{t_{0} \leqslant \tau \leqslant t}\left(\exp (-\tau) \frac{|x(\tau)|}{\mu(\tau)}\right), \\
& \forall t \in\left[t_{0}, t_{1}\right),
\end{aligned}
$$

which implies

$$
\sup _{t_{0} \leqslant \tau \leqslant t} \frac{|x(\tau)|}{\mu(\tau)} \leqslant 2 a\left(\beta\left(t_{0}\right)\left|x_{0}\right|\right), \quad \forall t \in\left[t_{0}, t_{1}\right) .
$$

Estimate (4.6) implies that the solution of (4.3) does not have a finite escape time and satisfies

$$
|x(t)| \leqslant 2 \mu(t) a\left(\beta\left(t_{0}\right)\left|x_{0}\right|\right), \quad \forall t \geqslant t_{0} .
$$

Thus system (4.3) is RFC with input $(\theta, d) \in D:=$ $\Omega \times B$. On the other hand estimates (4.2a) and (4.7) in conjunction with definition (4.4) provide the estimates

$$
\begin{aligned}
&|Y(t)| \leqslant \sigma\left(\beta\left(t_{0}\right)\left|x_{0}\right|, t-t_{0}\right) \\
&+\exp \left(-t_{0}\right) a\left(\beta\left(t_{0}\right)\left|x_{0}\right|\right), \quad \forall t \geqslant t_{0}, \\
&|Y(t)| \leqslant \sigma\left(\beta\left(t_{2}\right)\left|x\left(t_{2}\right)\right|, t-t_{2}\right) \\
&+ \frac{1}{2} \sup _{t_{2} \leqslant \tau \leqslant t}\left(\exp (-\tau) \frac{|x(\tau)|}{\mu(\tau)}\right), \\
& \forall t \geqslant t_{2} \geqslant t_{0} .
\end{aligned}
$$

Estimate (4.8a) guarantees that property P1 of Definition 3.1 holds for (4.3). Next we show that property P2 of Definition 3.1 holds for (4.3). Let arbitrary $\varepsilon>0, T \geqslant 0$ and $R \geqslant 0$. Clearly there exists $t_{2}:=$ $t_{2}(\varepsilon, T, R) \geqslant 0$ such that

$\exp \left(-t_{2}\right) a\left(\max _{0 \leqslant t \leqslant T} \beta(t) R\right) \leqslant \frac{\varepsilon}{2}$.

Consequently, estimate (4.7) implies that for all $\left|x_{0}\right| \leqslant R, t_{0} \in[0, T]$, and $t \geqslant t_{0}+t_{2}$ we have

$\frac{1}{2} \sup _{t_{0}+t_{2} \leqslant \tau \leqslant t} \exp (-\tau) \frac{|x(\tau)|}{\mu(\tau)} \leqslant \frac{\varepsilon}{2}$.

The above inequality in conjunction with (4.7) and (4.8b), give for all $\left|x_{0}\right| \leqslant R, t_{0} \in[0, T]$ :

$$
\begin{aligned}
|Y(t)| \leqslant & \sigma\left(2 \max _{0 \leqslant \tau \leqslant T+t_{2}} \beta(\tau) \mu(\tau)\right. \\
& \left.\times a\left(\max _{0 \leqslant \tau \leqslant T} \beta(\tau) R\right), t-t_{0}-t_{2}\right) \\
& +\frac{\varepsilon}{2}, \quad \forall t \geqslant t_{0}+t_{2} .
\end{aligned}
$$

Clearly there exists $t_{3}:=t_{3}(\varepsilon, T, R) \geqslant 0$ such that

$\sigma\left(2 \max _{0 \leqslant \tau \leqslant T+t_{2}} \beta(\tau) \mu(\tau) a\left(\max _{0 \leqslant \tau \leqslant T} \beta(\tau) R\right), t_{3}\right) \leqslant \frac{\varepsilon}{2}$.

The above inequality in conjunction with (4.9) implies that the property P2 of Definition 3.1 holds for the selection $\tau:=\tau(\varepsilon, T, R)=t_{2}(\varepsilon, T, R)+t_{3}(\varepsilon, T, R)$. The proof is complete.

Proposition 4.2. Suppose that in addition to the hypotheses of Proposition 4.1, $0 \in \mathfrak{R}^{n}$ is non-uniformly in time RGAS for system (4.1) with $v \equiv 0$. Then there exists a $C^{0}$ function $\gamma(t, s): \mathfrak{R}^{+} \times \mathfrak{R}^{+} \rightarrow \mathfrak{R}^{+}$, which is locally Lipschitz with respect to $s$, with $\gamma(t, \cdot) \in K_{\infty}$ 
for each $t \geqslant 0$, such that system (4.3) is non-uniformly in time RGAOS and zero is non-uniformly in time $R G A S$ for (4.3) with input $(\theta, d) \in D:=\Omega \times B$.

Proof. The proof relies on the following idea: it suffices to prove that system (4.1) satisfies an estimate of the form (4.2a) for the "extended" output $\tilde{Y}:=(x, H(t, x))$. Then Proposition 4.1 guarantees the existence of a $C^{0}$ function $\gamma(t, s): \mathfrak{R}^{+} \times \mathfrak{R}^{+} \rightarrow$ $\mathfrak{R}^{+}$, which is locally Lipschitz with respect to $s$, with $\gamma(t, \cdot) \in K_{\infty}$ for each $t \geqslant 0$, such that system (4.3) is non-uniformly in time RGAOS for the output $\tilde{Y}:=(x, H(t, x))$. This implies that system (4.3) is non-uniformly in time RGAOS and zero is non-uniformly in time RGAS for (4.3) with input $(\theta, d) \in D:=\Omega \times B$.

Since $0 \in \mathfrak{R}^{n}$ is non-uniformly in time RGAS for the "unforced" system (4.1) with $v \equiv 0$, then following exactly the same procedure with the proof of Lemma 2.7 in [10], we can prove that for every function $\mu(\cdot)$ of class $K^{+}$, there exists a $C^{\infty}$ map $V$ : $\mathfrak{R}^{+} \times \mathfrak{R}^{n} \rightarrow \mathfrak{R}^{+}$, functions $a_{i}(\cdot)(i=1, \ldots, 4)$ of class $K_{\infty}$ and $p(\cdot), \kappa(\cdot)$ of class $K^{+}$, such that

$a_{1}(|x|) \leqslant V(t, x) \leqslant a_{2}(p(t)|x|), \quad \forall(t, x) \in \mathfrak{R}^{+} \times \mathfrak{R}^{n}$

$$
\begin{aligned}
& \frac{\partial V}{\partial t}(t, x)+\frac{\partial V}{\partial x}(t, x) f(t, \theta, x, v) \\
& \leqslant-V(t, x)+\exp (-2 t) a_{3}\left(\frac{|x|}{\mu(t)}\right) a_{4}(\kappa(t)|v|), \\
& \quad \forall(t, \theta, x, v) \in \mathfrak{R}^{+} \times \Omega \times \mathfrak{R}^{n} \times \mathfrak{R}^{m} .
\end{aligned}
$$

Using inequalities $(4.10 \mathrm{a}, \mathrm{b})$ we obtain that the unique solution of (4.1) initiated from $x_{0} \in \mathfrak{R}^{n}$ at time $t_{0} \geqslant 0$ and corresponding to input $(\theta(\cdot), v(\cdot)) \quad \in$ $M_{\Omega} \times \mathrm{L}_{\mathrm{loc}}^{\infty}\left(\left[t_{0},+\infty\right)\right)$, satisfies the estimate for all $t \geqslant t_{0}$ :

$$
\begin{aligned}
& a_{1}(|x(t)|) \\
& \leqslant V(t, x(t)) \leqslant \exp \left(-\left(t-t_{0}\right)\right) V\left(t_{0}, x_{0}\right) \\
& \quad+\exp \left(-\left(t-t_{0}\right)\right) \sup _{t_{0} \leqslant \tau \leqslant t} a_{3}\left(\frac{|x(\tau)|}{\mu(\tau)}\right) \\
& \quad \times \sup _{t_{0} \leqslant \tau \leqslant t} a_{4}(\kappa(\tau)|v(\tau)|) .
\end{aligned}
$$

The above estimate in conjunction with estimate (4.2b) shows that there exist certain functions $\tilde{\sigma} \in$
$K L, \tilde{\rho} \in K_{\infty}, \tilde{\phi}, \tilde{\beta} \in K^{+}$such that for all $(\theta(\cdot), v(\cdot)) \in M_{\Omega} \times \mathrm{L}_{\mathrm{loc}}^{\infty}\left(\left[t_{0},+\infty\right)\right)$ it holds that

$$
\begin{aligned}
& |x(t)| \leqslant \tilde{\sigma}\left(\tilde{\beta}\left(t_{0}\right)\left|x_{0}\right|, t-t_{0}\right) \\
& \quad+\sup _{t_{0} \leqslant \tau \leqslant t} \tilde{\rho}(\tilde{\phi}(\tau)|v(\tau)|), \quad \forall t \geqslant t_{0} .
\end{aligned}
$$

The proof is complete since (4.12) in conjunction with estimate (4.2a) implies that there exist certain functions $\bar{\sigma} \in K L, \bar{\rho} \in K_{\infty}, \bar{\phi}, \bar{\beta} \in K^{+}$such that for all $(\theta(\cdot), v(\cdot)) \in M_{\Omega} \times \mathrm{L}_{\mathrm{loc}}^{\infty}\left(\left[t_{0},+\infty\right)\right)$ it holds that

$$
\begin{gathered}
|\tilde{Y}(t)|=|(x(t), Y(t))| \leqslant \bar{\sigma}\left(\bar{\beta}\left(t_{0}\right)\left|x_{0}\right|, t-t_{0}\right) \\
+\sup _{t_{0} \leqslant \tau \leqslant t} \bar{\rho}(\bar{\phi}(\tau)|v(\tau)|), \\
\forall t \geqslant t_{0} .
\end{gathered}
$$

\section{Conclusions}

The notions of non-uniform in time Robust Global Asymptotic Output Stability (RGAOS) and Robust Forward Completeness (RFC) are introduced. Characterizations for these notions were provided as well as links with non-uniform in time Robust Global Asymptotic Stability (RGAS). The results presented generalize corresponding characterizations given in the literature for the uniform in time case.

\section{Acknowledgements}

The author would like to thank Professor J. Tsinias for his comments and suggestions as well as the anonymous reviewers, who provided valuable comments.

\section{References}

[1] D. Angeli, E.D. Sontag, Forward completeness, unbounded observability and their Lyapunov characterizations, Systems Control Lett. 38 (4-5) (1999) 209-217.

[2] D. Angeli, E.D. Sontag, Y. Wang, A characterization of integral input-to-state stability, IEEE Trans. Automat. Control 45 (6) (2000) 1082-1096.

[3] A.V. Fillipov, Differential Equations with Discontinuous Right-Hand Sides, Kluwer Academic Publishers, Dordrecht, 1988.

[4] B. Ingalls, Y. Wang, On input-to-output stability for systems not uniformly bounded, Proceedings of NOLCOS, 2001.

[5] I. Karafyllis, Non-uniform stabilization of control systems, IMA J. Math. Control Inform. 19 (4) (2002) 419-444. 
[6] I. Karafyllis, Necessary and sufficient conditions for the existence of stabilizing feedback for control systems, IMA J. Math. Control Inform. 20 (1) (2003) 37-64.

[7] I. Karafyllis, J. Tsinias, Global stabilization and asymptotic tracking for a class of nonlinear systems by means of timevarying feedback, Internat. J. Robust Nonlinear Control 13 (6) (2003) 559-588.

[8] I. Karafyllis, J. Tsinias, A converse Lyapunov theorem for non-uniform in time global asymptotic stability and its application to feedback stabilization, SIAM J. Control Optim. 42 (3) (2003) 936-965.

[9] I. Karafyllis, J. Tsinias, Non-uniform in time stabilization for linear systems and tracking control for nonholonomic systems in chained form, Internat. J. Control 76 (15) (2003) $1536-1546$.

[10] I. Karafyllis, J. Tsinias, Non-uniform in time ISS and the small-gain Theorem, IEEE Trans. Automat. Control 49 (2) (2004) 196-216.
[11] J. Kato, A. Strauss, On the global existence of solutions and Lyapunov functions, Ann. Mat. Pura Appl. 77 (1967) 303-316.

[12] Y. Lin, E.D. Sontag, Y. Wang, A smooth converse Lyapunov theorem for robust stability, SIAM J. Control Optim. 34 (1996) 124-160.

[13] E.D. Sontag, Comments on integral variants of ISS, Systems Control Lett. 34 (1998) 93-100.

[14] E.D. Sontag, Y. Wang, Notions of input to output stability, Systems Control Lett. 38 (1999) 235-248.

[15] E.D. Sontag, Y. Wang, Lyapunov characterizations of inputto-output stability, SIAM J. Control Optim. 39 (2001) 226-249.

[16] A.R. Teel, L. Praly, A smooth Lyapunov function from a class KL estimate involving two positive semidefinite functions, ESAIM-Control Optim. Calculus Variations 5 (2000) 313-367. 